\title{
Adsorption Mechanism of Arsenic on Nanocrystalline Titanium Dioxide
}

\author{
Maria Pena, Xiaoguang Meng, George P. Korfiatis, Chuanyong Jing* \\ Center for Environmental Systems \\ Stevens Institute of Technology \\ Hoboken, NJ USA 07030
}

\section{Supporting Information}

Including 4 tables in 3 pages 
Table 1. Arsenic K-edge EXAFS fitting results of $\mathrm{As}(\mathrm{V})$ and $\mathrm{As}(\mathrm{III})$ reacted with $\mathrm{TiO}_{2}$.

\begin{tabular}{|c|c|c|c|c|c|c|}
\hline \multirow[b]{2}{*}{ Sample } & \multicolumn{3}{|c|}{ As-O shell } & \multicolumn{3}{|c|}{ As-Ti shell } \\
\hline & $\mathrm{R}(\AA)$ & $\mathrm{CN}$ & $\sigma^{2}\left(\AA^{2}\right)$ & $\mathrm{R}(\AA)$ & $\mathrm{CN}$ & $\sigma^{2}\left(\AA^{2}\right)$ \\
\hline $\mathrm{As}(\mathrm{V})$ & $1.69 \pm 0.01$ & $3.8 \pm 0.7$ & $0.0014 \pm 0.0015$ & $3.30 \pm 0.02$ & $2.5 \pm 1.0$ & $0.0065 \pm 0.0070$ \\
\hline As(III) & $1.77 \pm 0.01$ & $3.0 \pm 0.3$ & $0.0029 \pm 0.0074$ & $3.35 \pm 0.03$ & $1.7 \pm 0.8$ & $0.0105 \pm 0.0039$ \\
\hline
\end{tabular}

Table 2. FTIR band positions and assignments for As-O bonds in As(V) and As(III) dissolved species.

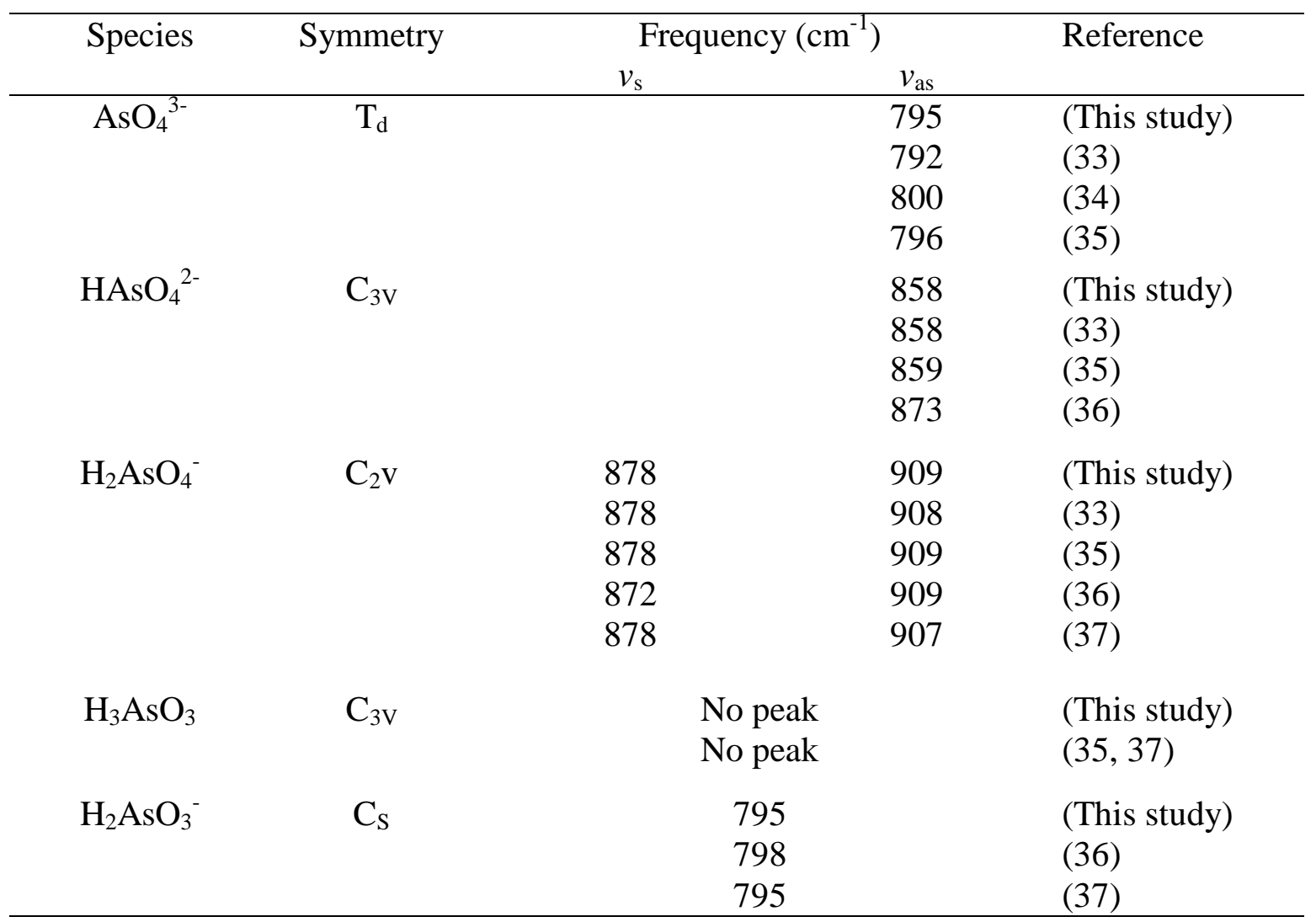


Table 3. FTIR band positions and assignments for adsorbed As(V) and As(III) species on $\mathrm{TiO}_{2}$ and other minerals.

\begin{tabular}{ccccc}
\hline Species & Symmetry & Frequency $\left(\mathrm{cm}^{-1}\right)$ & Description & Reference \\
\hline$A s(V)$ species & & & & \\
As(V)-TiO ${ }_{2}$ & $\mathrm{C}_{2 \mathrm{~V}}$ & 808 & $v(\mathrm{As}-\mathrm{OTi})$ & This study \\
& & 830 & $v(\mathrm{As}-\mathrm{O})$ & This study \\
$\mathrm{As}(\mathrm{V})-\mathrm{Fe}$ Oxide & & $817-824$ & $v(\mathrm{As}-\mathrm{OFe})$ & 37 \\
& & $854-861$ & $v(\mathrm{As}-\mathrm{O})$ & 37 \\
$\mathrm{As}(\mathrm{V})$-fresh Fe Oxide & $\mathrm{C}_{2 \mathrm{~V}}$ & 805 & $v(\mathrm{As}-\mathrm{OFe})$ & 31 \\
& & 875 & $v(\mathrm{As}-\mathrm{O})$ & 31 \\
$\mathrm{As}(\mathrm{V})-\mathrm{Ca}(\mathrm{OH})_{2}$ & & $798-812$ & $v(\mathrm{As}-\mathrm{OCa})$ & 31 \\
& & $885-905$ & $v(\mathrm{As}-\mathrm{O})$ & 31 \\
As-ettringite & $\mathrm{C}_{2} \mathrm{~V}$ & 808 & $v(\mathrm{As}-\mathrm{OCa})$ & 38 \\
& & $842-947$ & $v(\mathrm{As}-\mathrm{O})$ & 38 \\
As $(\mathrm{III})$ species & & & & \\
$\mathrm{As}(\mathrm{III})-\mathrm{TiO}{ }_{2}$ & $\mathrm{C}_{\mathrm{S}}$ & 780 & $v(\mathrm{As}-\mathrm{O})$ & This study \\
$\mathrm{As}(\mathrm{III})-\mathrm{Fe}(\mathrm{OH})_{3}$ & $\mathrm{Cs}$ & $789-794$ & $v(\mathrm{As}-\mathrm{O})$ & 36 \\
& $\mathrm{Cs}$ & 783 & $v(\mathrm{As}-\mathrm{O})$ & 37 \\
\hline
\end{tabular}

Table 4. Reactions and parameters used in the model calculations.

Aqueous reactions of $\mathrm{As}(\mathrm{V})$ and $\mathrm{As}(\mathrm{III})$

1) $\mathrm{HAsO}_{4}{ }^{-2}=\mathrm{AsO}_{4}^{-3}+\mathrm{H}^{+}$

2) $\mathrm{H}_{2} \mathrm{AsO}_{4}^{-}=\mathrm{AsO}_{4}^{-3}+2 \mathrm{H}^{+}$

3) $\mathrm{H}_{3} \mathrm{AsO}_{4}=\mathrm{AsO}_{4}^{-3}+3 \mathrm{H}^{+}$

4) $\mathrm{HAsO}_{3}^{-2}=\mathrm{AsO}_{3}^{-3}+\mathrm{H}^{+}$

5) $\mathrm{H}_{2} \mathrm{AsO}_{3}-=\mathrm{AsO}_{3}^{-3}+2 \mathrm{H}^{+}$

6) $\mathrm{H}_{3} \mathrm{AsO}_{3}=\mathrm{AsO}_{3}^{-3}+3 \mathrm{H}^{+}$

Surface protolysis

7) $\mathrm{TiOH}+\mathrm{H}^{+}=\mathrm{TiOH}_{2}^{+}$

8) $\mathrm{TiOH}=\mathrm{TiO}^{-}+\mathrm{H}^{+}$

$\mathrm{As}(\mathrm{V})$ and $\mathrm{As}(\mathrm{III})$ adsorption

9) $2 \mathrm{TiOH}+3 \mathrm{H}^{+}+\mathrm{AsO}_{4}{ }^{-3}=\mathrm{Ti}_{2} \mathrm{AsO}_{4}{ }^{-}+2 \mathrm{H}_{2} \mathrm{O}+\mathrm{H}^{+}$

10) $2 \mathrm{TiOH}+\mathrm{H}_{2} \mathrm{AsO}_{3}{ }^{-}=\mathrm{Ti}_{2} \mathrm{AsO}_{3}{ }^{-}+2 \mathrm{H}_{2} \mathrm{O}$ $\log \mathrm{K} \quad$ Ref.

$-11.60 \quad 23$

- 18.3523

$-20.60 \quad 23$

$-13.41 \quad 23$

$-25.52 \quad 23$

$-34.74 \quad 23$

$3.8 \quad 22$

$-7.822$

30.0 this study

-2.0 this study

Parameters used in the model:

$\mathrm{TiO}_{2}=0.2 \mathrm{~g} / \mathrm{L}$

Total absorbate $=1 \mathrm{mg} / \mathrm{L}$

Surface site $(\mathrm{SOH})$ density $=10.98$ sites $/ \mathrm{nm}^{2} \quad 22$

Site concentration $=1.2 \mathrm{mmol} / 1$

Surface Area $=329 \mathrm{~m}^{2} / \mathrm{g}$ 